\title{
Cardiac Implantable Electronic Device Reutilization: Battery Life of Explanted Devices at a Tertiary Care Center
}

\author{
LINDSEY GAKENHEIMER, B.S., JOSHUA ROMERO, M.D., TIMIR S. BAMAN, M.D., \\ DAN MONTGOMERY, B.S., CYDNI A. SMITH, B.A., HAKAN ORAL, M.D., \\ KIM A. EAGLE, M.D., and THOMAS CRAWFORD, M.D. \\ From the Division of Cardiovascular Medicine, Department of Medicine, University of Michigan, Ann Arbor, \\ Michigan
}

Introduction: Prior studies have suggested that pacemaker reuse may be a reasonable alternative to provide device therapy in the low- and middle-income countries. We studied explant indications and remaining battery life of cardiac implantable electronic devices (CIEDs) at a tertiary medical center.

Methods and Results: We conducted a retrospective review of all CIEDs extracted at the University of Michigan between 2007 and 2011. Devices were considered reusable if battery longevity was $\geq 48$ months or $>75 \%$ battery life was remaining; there was no evidence of electrical malfunction, and they were not under advisory or recall. Eight hundred and one CIEDs were explanted: Medtronic (MDT [Medtronic Inc., Minneapolis, MN, USA]; 454), Boston Scientific (BS [Boston Scientific Corp., Natick, MA, USA])/Guidant (GDT; 255 [Guidant Corp., St. Paul, MN, USA]), St. Jude Medical (SJM; 73 [St. Paul, MN, USA]), and Biotronik (BTK; 15 [Biotronik GmBH, Berlin, Germany]). After eliminating devices explanted for elective replacement indicator (ERI, 541), 51.9\% of pacemakers (41/79), 54.2\% of implantable cardioverterdefibrillators (ICDs) (64/118), and 47.6\% of cardiac resynchronization therapy and defibrillation (CRT-D) devices (30/63) had sufficient battery life and no evidence of electrical malfunction to be considered for reuse. A logistic regression analysis found that the indications for device removal independently predicted reusability: upgrade to an ICD (odds ratio [OR] 162.8, $P<0.001$ ) or CRT-D (OR 63.8, $P<0.001$ ), infection (OR 110.7, $P<0.001$ ), heart transplantation or left ventricular assist device placement (OR 56.6, $P<$ 0.001 ), and device removal at patient's request (OR 115.4, $P<0.001$ ).

Conclusion: The majority of explanted CIEDs for reasons other than ERI have an adequate battery life and, if proven safe, may conceivably be reutilized for basic pacing in underserved nations where access to this life-saving therapy is limited. (PACE 2014; 37:569-575)

pacing, defibrillation - ICD

Pacemaker and implantable cardioverterdefibrillator (ICD) use has been shown to reduce morbidity and mortality in select patients with cardiovascular disease. ${ }^{1,2}$ Cardiovascular disease is not only the leading cause of death in the high-income countries, but also in the developing world, where it causes twice as many deaths as human immunodeficiency virus (HIV), malaria, and tuberculosis combined. ${ }^{3}$ In some lowand middle-income countries (LMIC), infectious diseases such as Chagas or African Sleeping Sickness add to the bradyarrhythmia burden. ${ }^{4,5}$

None of the authors report a conflict of interest.

Address for reprints: Thomas Crawford, M.D., Cardiovascular Center, The University of Michigan, 1500 East Medical Center Drive, SPC 5853, Ann Arbor, MI 48109-5853. Fax: 734-9367026; e-mail: thomcraw@umich.edu

Received December 29, 2012; revised October 14, 2012; accepted October 20, 2013.

doi: 10.1111/pace.12321
Unfortunately, cost barriers prevent many patients in the developing countries from benefiting from cardiac implantable electronic devices (CIEDs). The pacemaker and defibrillator implantation rates are dramatically lower in LMIC. For instance, in 2009, Peru and Bangladesh had implantation rates of 30 and five pacemakers per million people, respectively, compared with 767 implants per million population in the United States and 782 in France. ${ }^{6}$ The low implantation rates in LMIC reflect poor access to electrophysiological care due to a host of factors, one being the economic conditions.

CIED reutilization has been proposed as a means to eliminate the disparity in pacemaker therapy between the industrialized world and LMIC. $^{7}$ This study explores the potential of harvesting "good" devices at the time of CIED removal for clinical indications. We examined the reasons for explantation as well as the remaining battery life of CIEDs explanted at a tertiary medical center. Any device with an adequate battery

(C)2013 Wiley Periodicals, Inc. 
life and free from known malfunction could potentially be reutilized in LMIC patients who otherwise may not have access to this potentially life-saving therapy.

\section{Methods}

A retrospective chart review was performed on all CIEDs explanted at the University of Michigan from January 2007 until January 2011. Device parameters such as make, model, serial number, battery voltage, percent pacing, pacing thresholds, lead impedances, and time to charge, when applicable, were collected. Parameters were obtained at the time of, or within 3 months prior to, CIED explantation. Patient demographics and clinical data were also collected, including age, sex, and indication for device implantation and explantation. Criteria for a "reusable" device were $>75 \%$ of the original battery life or $\geq 48$ months remaining and freedom from device recall or failure. Battery longevity of Medtronic Inc. (Minneapolis, MN, USA) ICDs and pacemakers utilizing an ICD interrogation platform was estimated by using the date of implant and battery voltage as provided by Medtronic Technical Services (personal communication, January 26, 2010). Battery longevity of some Guidant/Boston Scientific devices (Guidant Corp., St. Paul, MN, USA; Boston Scientific, Natick, MA, USA) are expressed in descriptive terms: BOL (beginning of life)/GOOD/OKAY/ERI (elective replacement indicator) and as a "fuel gauge." Voltages for these devices were not included in the analysis. All of the BOL devices were considered reusable. Any device that was GOOD or OKAY was considered reusable only if the fuel gauge showed $75 \%$ or greater of the original battery life.

\section{Statistical Analysis}

Continuous variables were compared using the $t$-test, while categorical variables were compared using the $\chi^{2}$ or Fisher exact test, where appropriate. Logistic regression analysis was performed to determine predictors of a device being "reusable." Analyses were performed using SPSS (15.0) for Windows (IBM Corp., Armonk, NY, USA). $\mathrm{P}<0.05$ indicated statistical significance.

\section{Device Data}

During the study period, 2,452 device procedures were performed at the University of Michigan. A total of 801 devices were explanted. The manufacturers of the explanted generators were Medtronic (MDT; 454), Boston Scientific/Guidant (BS)/(GDT; 255), St. Jude Medical
(SJM; 73 [St. Paul, MN, USA]), and Biotronik (BTK; 15 [Biotronik GmBH, Berlin, Germany]). The name of the manufacturer of four devices could not be identified. Five hundred and forty-one devices were explanted for ERI and 260 for all other indications. Of the 801 devices, $13.2 \%(41 / 311)$ of pacemakers, $21.0 \%(64 / 305)$ of ICDs, and $16.2 \%$ (30/185) of cardiac resynchronization therapy and defibrillation devices (CRT-Ds) had sufficient battery life and no evidence of malfunction. After eliminating the 541 devices explanted for ERI, $51.9 \%$ of pacemakers (41/79), $54.2 \%$ of ICDs (64/118), and $47.6 \%$ of CRT-Ds (30/63) met the criteria for reuse. Figure 1 shows the numbers of reusable and nonreusable CIEDs according to the device type and manufacturer among explanted non-ERI devices in the study.

Figure 2 compares the average length of time, in months, that nonreusable and reusable devices remained inside their owner. Devices deemed reusable were, on average, 4.5 years younger than the nonreusable devices (average age of reusable devices: $17.78 \pm 1.77$ months; average age of nonreusable devices: $72.09 \pm 2.26$ months, $\mathrm{P}=$ 0.003 ).

A measure of the percent pacing found that reusable devices had paced less than $50 \%$ of the time (percent atrial pacing: $43.84 \% \pm 6.55 \%$; percent ventricular pacing: $49.43 \% \pm 5.20 \%$ ). The average pacing thresholds in the reusable devices were fairly low (atrial pacing threshold: $1.01 \pm 0.14 \mathrm{~V}$ at $0.45 \pm 0.02 \mathrm{~ms}$; right ventricular pacing threshold: $1.15 \pm 0.10 \mathrm{~V}, 0.48 \pm 0.02$ $\mathrm{ms}$; left ventricular pacing threshold: $2.21 \pm$ $0.43 \mathrm{~V}, 0.66 \pm 0.10 \mathrm{~ms}$ ). Average impedance measurements were $520.9 \pm 41.4 \mathrm{~V}$ in the atrium, $570.4 \pm 34.2 \mathrm{~V}$ in the right ventricle, and $569.2 \pm$ $53.0 \mathrm{~V}$ in the left ventricle. Table I lists the indications for CIED explantation. Devices that were explanted for infection, upgrade to a high voltage and/or CRT device, lead failure, heart transplant, or left ventricular assist device (LVAD) implantation were more likely to contain sufficient battery life for potential reuse. A logistic regression analysis determined that the following indications for explantation were independent predictors for a device being reusable: infection, an upgrade to an ICD or CRT-D, heart transplant/LVAD implantation, patient request, and lead failure (Table II). SJM or BS/GDT devices were less likely to meet the criteria for reuse, although the finding for BS/GDT devices was not statistically significant.

\section{Patient Demographics}

Younger patients were more likely to have a reusable device $(56.20 \pm 1.49$ and $61.21 \pm$ 0.766 years for patients with a reusable and 


\section{Type of Device}

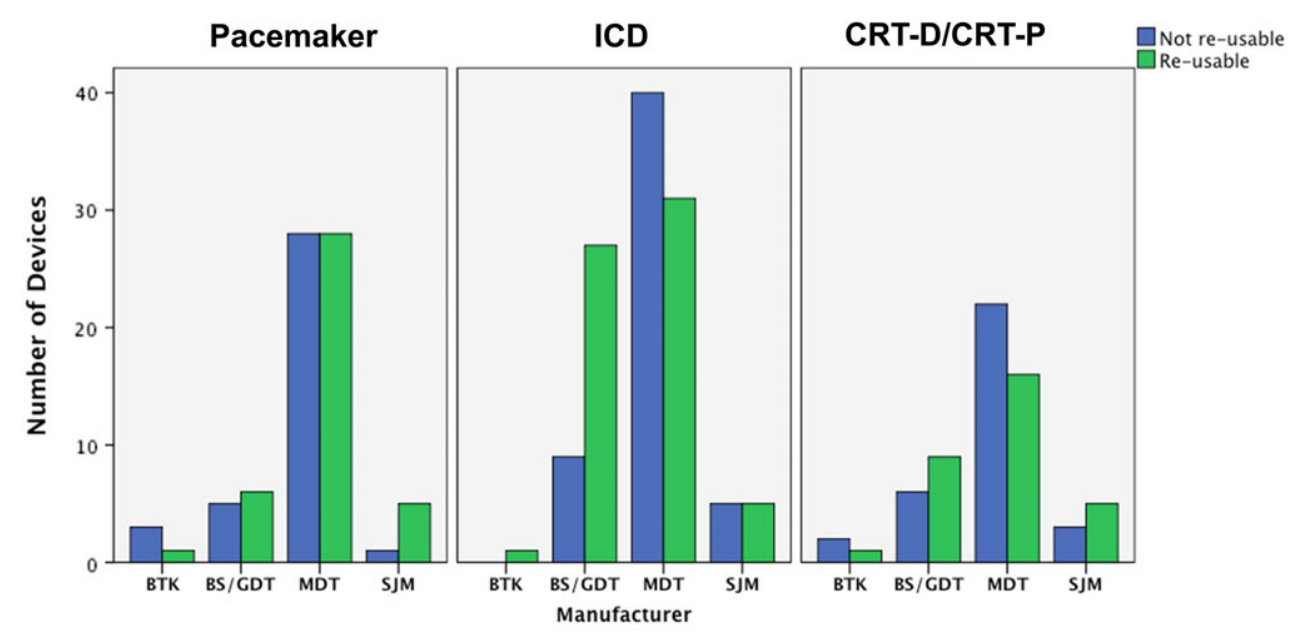

Figure 1. The number of reusable and nonreusable CIEDs according to the device type and manufacturer among explanted non-ERI devices in the study. BS/GDT = Boston Scientific/Guidant; BTK = Biotronik; $C R T-D=$ cardiac resynchronization therapy-defibrillator; $C R T-P=$ cardiac resynchronization therapy—pacemaker; ERI = elective replacement indicator; $I C D=$ implantable cardioverter-defibrillator; $M D T=$ Medtronic; SJM = St. Jude Medical.

\section{Type of Device}

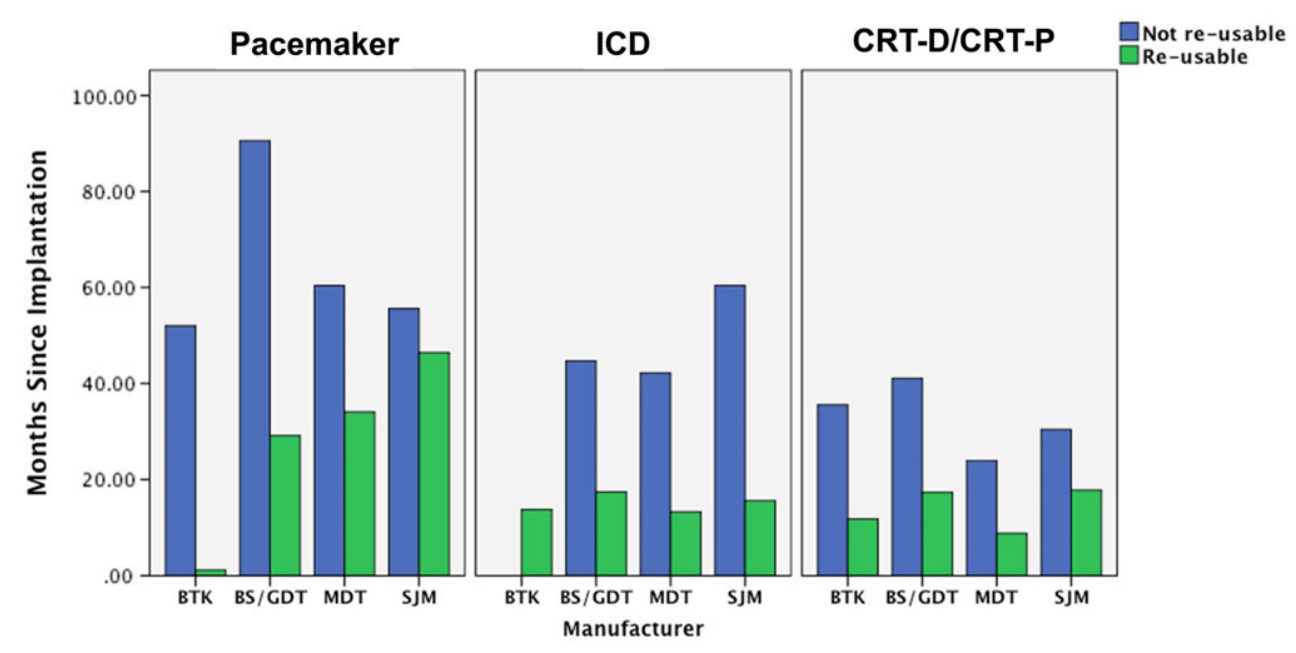

Figure 2. The length of time, in months, from initial CIED implantation to generator removal for non-ERI devices in the study. Abbreviations as in Figure 1.

nonreusable device, respectively, $\mathrm{P}=0.007$ ). Males were 3.5 times mores likely to have a reusable device than females $(75.8 \%$ of males vs $24.2 \%$ of females, $\mathrm{P}=0.027)$. Tables III and IV list the indications for ICDs and pacemakers, respectively. Patients who had an ICD for primary prevention of sudden cardiac death (nonischemic cardiomyopathy [NICM] or ischemic cardiomyopathy [ICM]) were more likely to have a reusable device than patients with initial ICD indication of secondary prevention, although this did not reach statistical significance $(\mathrm{P}=0.058)$. There was no difference in the indications for pacemakers in the reusable versus the nonreusable devices.

\section{Discussion}

Cardiovascular disease is the leading cause of death in the developing world. ${ }^{3}$ It is estimated 
Table I.

List of the Reasons for Explantation

\begin{tabular}{lcrr}
\hline Indication for Explantation & Useable Devices $(\mathbf{n}=\mathbf{1 3 5})$ & Nonuseable Devices (n=666) & P-Value \\
\hline ERI & $0(0.0 \%)$ & $541(81.2 \%)$ & $<0.001$ \\
Infection & $43(31.9 \%)$ & $19(2.4 \%)$ & $<0.001$ \\
Upgrade to ICD & $13(9.6 \%)$ & $7(1.0 \%)$ & $<0.001$ \\
Upgrade to CRT-D & $27(20.0 \%)$ & $33(5.0 \%)$ & $<0.001$ \\
Upgrade to dual-chamber pacemaker & $1(0.7 \%)$ & $0(0.0 \%)$ & 0.024 \\
Upgrade to CRT-pacemaker & $3(2.2 \%)$ & $1(0.1 \%)$ & 0.002 \\
Heart transplant/ LVAD implantation & $25(18.5 \%)$ & $19(2.9 \%)$ & $<0.001$ \\
Device failure & $0(0.0 \%)$ & $16(2.4 \%)$ & 0.073 \\
Lead failure & $13(9.6 \%)$ & $26(3.9 \%)$ & 0.004 \\
Device recall & $0(0.0 \%)$ & $2(0.3 \%)$ & 0.529 \\
Patient request & $6(4.4 \%)$ & $6(0.9 \%)$ & 0.002
\end{tabular}

Devices explanted for reasons of an infection, an upgrade to a different device, a heart transplantation/LVAD implantation, a lead failure, or because the patient no longer wants the device had a battery life $\geq 48$ months. These devices were also free of malfunctions and thus could potentially be reutilized. CRT-D = cardiac resynchronization therapy-defibrillator; ERI = elective replacement indicator; ICD = implantable cardioverter-defibrillator; LVAD = left ventricular assist device.

Table II.

Results from a Logistic Regression Analysis, Which Was Used to Determine the Independent Predictors for Devices That Could be Reutilized

\begin{tabular}{lcc}
\hline Variable & Odds Ratio (OR) & P-Value \\
\hline Device age (months) & 0.929 & $<0.001$ \\
Patient age at explantation & 0.998 & 0.832 \\
Explanted for infection & 110 & $<0.001$ \\
Explanted for upgrade to ICD & 163 & $<0.001$ \\
Explanted for upgrade to CRT-D & 63.8 & $<0.001$ \\
Explanted for heart transplant/ LVAD implantation & 56.6 & $<0.001$ \\
Patient requested explantation & 115 & $<0.001$ \\
Explanted for lead failure & 40.6 & $<0.001$ \\
SJM device & 0.216 & 0.012 \\
GDT/BS device & 0.251 & 0.252
\end{tabular}

Devices explanted for an infection, a device upgrade, heart transplant/LVAD implant, at the request of the patient, or lead failures are overwhelmingly likely to meet the criteria for reuse. St. Jude Medical (SJM) devices were more likely to be reusable. GDT/BS = Guidant/Boston Scientific. Other abbreviations as in Table I.

that about one million people die each year in the world due to lack of access to pacemaker therapy. The main finding of this study is that nearly $50 \%$ of devices explanted in a tertiary medical center for reasons other than ERI appear free from malfunctions and have $>75 \%$ of the original battery life or at least 4 years of the battery life remaining. These devices could potentially be reutilized in patients in LMICs, where access to bradyarrhythmia therapy is extremely limited. The yield of reusable devices during non-ERIrelated device procedures $(\sim 50 \%)$ is substantially higher than among devices reclaimed postmortem, where the yield of adequate battery life was only $21 \%$ for pacemakers and ICDs and $30 \%$ for CRTDs. ${ }^{8}$ Should pacemaker or ICD reuse become widely embraced, these findings would inform refurbishing centers that devices obtained during non-ERI-related device procedures should be a major focus of their efforts. According to the National Cardiovascular Data Registry (NCDR), 63,522 devices, representing about $41 \%$ of all ICD generator procedures, were explanted annually in years 2010-2012 (Personal Communication, Paul Heidenreich, M.D., M.S. member, NCDR Science \& Quality Oversight Committee, NCDR ${ }^{\circledR}$ 
Table III.

List of Patients' Indications for Their ICD/CRT-D

\begin{tabular}{lccc}
\hline Indication for ICD/CRT-D & $\begin{array}{c}\text { Useable ICD/CRT-D } \\
(\mathbf{n}=\mathbf{9 4})\end{array}$ & $\begin{array}{c}\text { Nonuseable ICD/CRT-D } \\
(\mathbf{n}=\mathbf{3 9 6})\end{array}$ & P-Value \\
\hline Primary prevention of SCD & $73(77.6 \%)$ & $268(67.7 \%)$ & 0.058 \\
Secondary prevention of SCD & $15(16.0 \%)$ & $91(23.0 \%)$ & 0.224 \\
Other & $6(6.4 \%)$ & $37(9.3 \%)$ & 0.806 \\
\hline
\end{tabular}

Devices from patients with primary prevention indication were more likely to be suitable for reuse. SCD = sudden cardiac death. Other abbreviations as in Table I.

Table IV.

List of Patients' Indications for Their Pacemaker

\begin{tabular}{lcrc}
\hline Indication for Pacemaker & Useable Pacemaker $(\mathbf{n}=\mathbf{4 1})$ & Nonuseable Pacemaker $(\mathbf{n}=\mathbf{2 7 0})$ & P-Value \\
\hline Sick sinus syndrome & $16(39.0 \%)$ & $99(36.7 \%)$ & 0.777 \\
Atrioventricular block & $23(56.1 \%)$ & $150(55.6 \%)$ & 0.999 \\
Syncope & $1(2.4 \%)$ & $7(2.6 \%)$ & 0.715 \\
Carotid sinus hypersensitivity & $0(0.0 \%)$ & $8(3.0 \%)$ & 0.318 \\
Other & $1(2.4 \%)$ & $6(2.2 \%)$ & 0.632 \\
\hline
\end{tabular}

There was no significant correlation between patients' indication and the reusability of their device.

ICD Registry ${ }^{\mathrm{TM}}$ October 11, 2012). If the yield of reusable ICD harvesting across the United States is similar to that at the University of Michigan, potentially as many as 10,000-13,000 devices with adequate battery life could be obtained each year for donation.

In our series, the largest number of reusable devices was due to infection followed by upgrade to CRT-D and heart transplantation or LVAD implantation. The rates of adequate battery life are similar among pacemakers, ICDs, and CRTDs $(52 \%, 54 \%$, and $48 \%$, respectively). This is unlike in the setting of postmortem device collection, where CRT-D had substantially greater remaining longevity than pacemakers and ICDs. ${ }^{8}$ The difference is likely explained by higher disease acuity of patients in whom care is escalated, as in upgrades to high voltage and resynchronization therapy. The extraction indications associated with the greatest chance of adequate battery are upgrade to ICD, patient request for device removal, and infection (odds ratio 163, 115 , and 110, respectively). High battery voltages commonly found in these devices would likely translate into many years of use in patients with bradyarrhythmia. This would especially be the case for patients receiving an ICD or CRT-D device, for the defibrillator function in these donated devices would not be utilized.
Devices manufactured by SJM and BS/GDT were less likely to meet the criteria for reuse in this study, as indicated by the logistic regression analysis, although this did not reach statistical significance for BS/GDT. The reason for this is not clear. It is possible that some patients with Medtronic ICD generators underwent replacements in advance of reaching ERI due to concern about the Fidelis lead integrity.

Infected devices may be well suited for device reutilization because the vast majority of those devices are explanted within a few weeks of implantation. ${ }^{9}$ Prior studies have shown that infected devices, when properly handled and sterilized, can be reutilized without significant complications. ${ }^{10,11}$ Recently implanted devices may have 10 - to 12-year projected longevity, highlighting the current waste of this costly resource. Infected devices must undergo a rigorous sterilization process. Further studies are needed to prospectively examine safety of device reuse from patients with device-related infections.

A recent study described an experience with reprocessed devices obtained antemortem in 17 patients. ${ }^{12}$ Ten devices had been removed for device upgrades and seven for infections. During mean follow-up period of $68 \pm 38$ months, no device infection or device malfunction was observed. A meta-analysis compared the safety of 
new and reutilized devices in 2,270 patients across 18 trials. $^{13}$ It found that, at 10 years, the overall mortality of patients who received refurbished devices was no higher than the mortality of patients with new devices. Moreover, the infection rate of the refurbished devices was no higher than that with the new devices, around $1.97 \%$. The malfunction rate was higher in refurbished devices, but it was still less than $1 \%$. A rigorous, large prospective trial of safety and efficacy of device reuse is needed before device reuse can gain wider acceptance as a means of charitable support for patients in LMIC who cannot afford a new pacemaker.

One other issue must be considered, as it relates to device harvesting during non-ERI device change-out and upgrade procedures. Who is the owner the device? Is it the patient, the manufacturer, or the insurance scheme, which paid for the device-whether it be private insurance or Medicare/Medicaid? One can postulate that the patient is the owner of the device. Even if a third-party payment is considered, those funds are raised through premiums and taxes, thus indirectly paid by the patient.

The National Institutes of Health appear to support the idea of patient ownership in a consensus program regarding medical device ownership. ${ }^{14}$ It seems intuitive that patient consent is required prior to donation. Previous research has found that about $90 \%$ of patients with a CIED are willing to donate them if provided the chance. ${ }^{8,15}$ The manufacturers encourage device return for quality assurance and proper disposal. Centers of excellence interested in device reuse could return devices, which do not meet criteria for reuse, to the manufacturers to assist in the process of device analysis and proper disposal, especially of the lithium battery component. Such a collaborative approach would allow for donation of refurbished devices to needy patients in the LMICs, as well as foster quality assurance and proper device disposal.

It is important to note that CIED collection and evaluation is only the first step in a long and arduous journey to making device reuse a reality. A validated cleaning and sterilization protocol is needed to provide patients and physicians with some degree of confidence that the risk of infection would not be significantly higher than with brand new pacemaker implants. Additionally, given the unusual handling of the devices, functionality testing must also be developed and implemented to assure the integrity of the CIED prior to reimplantation. A party with experience in medical device reprocessing may be needed to carry out key processes, and to engage with regulatory bodies in the United States (i.e., U.S. Food and Drug Administration) and the recipient countries. Although implantation of reprocessed devices goes on in many countries, it often happens without the explicit sanctioning by the health authorities. A nonprofit organization may be created to raise funds necessary to collect, reprocess, and distribute previously used CIEDs. An additional role of the nonprofit would be to assess qualification and safety record of potential implanting centers and physicians, and to maintain a database of all devices received and distributed. In addition to cost of handling and shipping, depending on the local infrastructure and resources, partnerships with charitable organizations may help to cover the expense of the leads and disposables if these are not available at the implantation center. The implantation centers' role would be to assess the indications for CIED and patient's financial status, safely implant the devices, and provide follow-up monitoring and detailed documentation on all devices. In short, a great many legal and logistical obstacles will have to be overcome through a collaborative effort with multiple parties in order to offer safe device implantation in patients with greatest need.

Project My Heart Your Heart is committed to addressing these important issues by furthering research in safe device reuse and developing the necessary partnerships to allow implementation of this concept. This organization accepts devices from funeral homes, crematories, hospitals, and family members of deceased device patients. Potential donors can go to website www.myheartyourheart.org to enter their contact information and shipping address. This organization sends a prepaid self-addressed flat-rate United States Postal Service envelope containing biohazard bags for each device, instructions on device removal, and how to return devices to our center. We believe that the development of standardized protocols for collection, evaluation, and resterilization will make CIED reuse safe and efficacious.

\section{Limitations}

Limitations of this study include the method used to obtain our results. Direct battery measurement with a load attached to the pacemaker was not performed; rather, telemetry measurements collected in the device clinic just prior to generator change were analyzed. Direct battery measurement would provide a more reliable battery longevity assessment. Additionally, since device parameters were collected within 3 months prior to explanation, they may slightly overestimate the true battery life potential. 


\section{Conclusion}

A large percentage of CIEDs explanted at tertiary care centers have ample battery life remaining. These devices could be reutilized to patients in developing nations who cannot afford a device. To ensure that devices can safely be

\section{References}

1. Zwanziger J, Hall WJ, Dick AW, Zhao H, Mushlin AI, Hahn RM, Wang H, et al. The cost effectiveness of implantable cardioverterdefibrillators: Results from the Multicenter Automatic Defibrillator Implantation Trial (MADIT)-II. J Am Coll Cardiol 2006; 47:23102318.

2. Lamas GA, Knight JD, Sweeney MO, Mianulli M, Jorapur V, Khalighi $\mathrm{K}$, Cook JR, et al. Impact of rate-modulated pacing on quality of life and exercise capacity: Evidence from the Advanced Elements of Pacing Randomized Controlled Trial (ADEPT). Heart Rhythm 2007; 4:1125-1132.

3. Gaziano TA. Reducing the growing burden of cardiovascular disease in the developing world. Health Affair 2007; 26:13-24.

4. Maudlin IHP, Miles MA. The Trypanosomiases. Cambridge, MA, CABI Publishing, 2004.

5. Blum JA, Zellweger MJ, Burri C, Hatz C. Cardiac involvement in African and American trypanosomiasis. Lancet Infect Dis 2008; 8:631-41.

6. Mond HG, Proclemer A. The 11th world survey of cardiac pacing and implantable cardioverter-defibrillators: Calendar year 2009-A World Society of Arrhythmia's project. Pacing Clin Electrophysiol 2011; 34:1013-1027.

7. Baman TS, Kirkpatrick JN, Romero J, Gakenheimer L, Romero A, Lange DC, Nosowsky R, et al. Pacemaker reuse: An initiative to alleviate the burden of symptomatic bradyarrhythmia in impoverished nations around the world. Circulation 2010; 122:1649-1656.

8. Baman TS, Crawford T, Sovitch P, Meier P, Sovitch N, Gakenheimer L, Kirkpatrick J, et al. Feasibility of postmortem device acquisition for potential reuse in underserved nations. Heart Rhythm 2012; 9:211-214. reused, a clinical trial should be performed, testing the safety of the refurbished devices immediately after implantation and at 6-month follow-up. If successful, device reutilization has the potential to save thousands of patients currently perishing due to inaccessibility to a currently wasted resource.

9. Baddour LM, Epstein AE, Erickson CC, Knight BP, Levison ME, Lockhart PB, Masoudi FA, et al. Update on cardiovascular implantable electronic device infections and their management: A scientific statement from the American Heart Association. Circulation 2010; 121:458-477.

10. Panja MSC, Kumar S, Kar AK, Mitra S, Sinha DP, Chatterjee A, Roy S, et al. Reuse of pacemaker. Indian Heart J 1996; 48:677680 .

11. Mansour KA, Kauten JR, Hatcher CR, Jr. Management of the infected pacemaker: Explantation, sterilization, and reimplantation. Ann Thorac Surg 1985; 40:617-619.

12. Hasan R, Ghanbari H, Feldman D, Menesses D, Rivas D, Zakhem NC, Duarte C, et al. Safety, efficacy, and performance of implanted recycled cardiac rhythm management (CRM) devices in underprivileged patients. Pacing Clin Electrophysiol 2011; 34:653658.

13. Baman TS, Meier P, Romero J, Gakenheimer L, Kirkpatrick JN, Sovitch P, Oral H, et al. Safety of pacemaker reuse: A metaanalysis with implications for underserved nations. Circ Arrhythm Electrophysiol 2011; 4:318-323.

14. US Department of Health and Human Services NIoH. Improving Medical Implant Performance through Retrieval Information: Challenges and Opportunities. NIH Technology Assessment Conference Summary. Bethesda, MD, National Institutes of Health, Consensus Development Program, 2000.

15. Kirkpatrick JN, Ghani SN, Burke MC, Knight BP. Postmortem interrogation and retrieval of implantable pacemakers and defibrillators: A survey of morticians and patients. J Cardiovasc Electrophysiol 2007; 18:478-482. 